\title{
ALGEBRAIC REPRESENTATION OF A CLASS OF HOMOGENOUS STEINER QUADRUPLE SYSTEMS
}

\author{
Lidija Goračinova-Ilieva ${ }^{1}$, Emilija Spasova Kamčeva ${ }^{2}$
}

\begin{abstract}
A Steiner system $S(t, k, v)$ is a pair $(Q, B)$ of $v$-element set $Q$ and a collection $B$ of its $k$-element subsets (blocks), such that every $t$-element subset of $Q$ is contained in exactly one block. Systems $S(2,3, v)$ are Steiner triple systems (STS) and their algebraic representatives are the idempotent totally symmetric quasigroups. Steiner quadruple systems ( $S Q S)$ are systems $S(3,4, v)$, represented by the idempotent totally symmetric ternary quasigroups.

For $S Q S(Q, B)$ and $a \in Q$, by taking the set $Q \backslash\{a\}$ and the blocks $\{\{x, y, z\}\{\{x, y, z, a\} \in B\}$, a derived triple system is obtained. An SQS is called homogenous if all of its derived triple systems are isomorphic.

In this paper sufficient conditions for $S Q S$ to be homogenous are given, resulting with an algebraic representation of one class of homogenous quadruple systems.
\end{abstract}

\section{INTRODUCTION}

A Steiner system $S(t, k, v)$ is a pair $(Q, B)$, where $Q$ is a $v$-element set and $B$ is a collection of its $k$-element subsets (called blocks) with the property that every $t$-element subset of $Q$ is contained in a unique block of $B$. Systems $S(2,3, v)$ and $S(3,4, v)$ are called Steiner triple system (STS) and Steiner quadruple system $(S Q S)$ respectively.

2010 Mathematics Subject Classification. Primary 51E10; Secondary 05E15. Key words and phrases. Steiner quasigroup, derived triple system, homogenous quadruple system, variety 
There is a natural connection between $\operatorname{SQS}(v)$ and $\operatorname{STS}(v-1)$. Let $(Q, B)$ be an $\operatorname{SQS}(v)$ and choose an arbitrary element $a \in Q$. Let $B_{a}$ be the collection of the 3-element subsets of $Q \backslash\{a\}$ which is obtained by selecting all of the blocks of $B$ containing the element $a$, and then excluding this element from them. Then the pair $\left(Q \backslash\{a\}, B_{a}\right)$ is an $\operatorname{STS}(v-1)$. Such a Steiner triple system is called a derived triple system (DTS) of the quadruple system $(Q, B)$. The problem whether or not every STS is a DTS of some quadruple system is open.

Woolhouse in 1844 [12] posed the question: for which integers $t, k$, and $v$, does an $S(t, k, v)$ exist? Up to the present time, this problem is also unsolved in general. However, several partial answers are given. Three years later, Kirkman [7] showed that $S T S(v)$ exists if and only if $v \equiv 1$ or $3(\bmod 6)$, and constructed systems $S\left(3,4,2^{n}\right)$, for every $n$. During the late $19^{\text {th }}$ and early $20^{\text {th }}$ century very much was written on the subject of $S T S$, and very little on $S Q S$. Hanani [4] proved that the necessary condition $v \equiv 2$ or $4(\bmod 6)$ for the existence of an $S Q S$ of order $v$ is also sufficient, by induction and using six recursive constructions. However, the most extensive study of Steiner systems probably was done in the 70 s and the 80 s of the last century, concerning the various constructions of a single system and classes of certain type, isomorphism problems, groups of automorphisms, classifications and enumerations, embedings and partial systems, as well as their applications. The development of the computers played significant role, especially in the past thirty years.

Steiner triple and quadruple systems possess "algebraic twins". Given an $S T S(v)(Q, B)$, one can define a binary operation $*$ on $Q$ by $a * b=c$ whenever $\{a, b, c\} \in B$, and $a * a=a$. As there is a unique triple in $B$ containing two distinct elements, this operation is well defined. The groupoid $(Q, *)$ belongs to the variety determined by the identities:

$$
\begin{aligned}
& x \cdot x=x \\
& x \cdot y=y \cdot x \\
& x \cdot(x \cdot y)=y .
\end{aligned}
$$

Its members are idempotent totally symmetric quasigroups, also known as Steiner quasigroups, since there is a two-way relationship between such 
quasigroups and Steiner triple systems. Namely, if $(Q, \cdot)$ is a $v$-element Steiner quasigroup, then the sets $\{a, b, a \cdot b\}, a, b \in Q, a \neq b$, are precisely the blocks of an $\operatorname{STS}(v)$.

A similar correspondence exists between Steiner quadruple systems and idempotent totally symmetric ternary quasigroups (Steiner 3-quasigroups). They are defined by the following identities:

$$
\begin{aligned}
& f(x, x, y)=y \\
& f(x, y, z)=f(x, z, y)=f(y, x, z) \\
& f(x, y, f(x, y, z))=z .
\end{aligned}
$$

If $(Q, B)$ is an $S Q S$, then the ternary operation $f$ on $Q$ defined by the rules $f(a, a, b)=f(a, b, a)=f(b, a, a)=b \quad$ and $\quad f(a, b, c)=d \quad$ if and only if $\{a, b, c, d\} \in B$ is well defined and satisfies the above identities. On the other hand, if $(Q, f)$ is a finite Steiner 3-quasigroup, then $(Q, B)$ is an $S Q S$, for $B$ consisting of the sets $\{a, b, c, f(a, b, c)\}$, where $a, b$ and $c$ are distinct elements of $Q$ (see [1]). For the quadruple system $(Q, B)$ corresponding to the Steiner 3-quasigroup $(Q, f)$, we will say that it is induced by $(Q, f)$. Note that for $a \in Q$, the $D T S\left(Q \backslash\{a\}, B_{a}\right)$ of $(Q, B)$ has an algebraic equivalent $(Q \backslash\{a\}, \cdot)$ whose operation can be defined by the ternary operation $f$, according to the rules

$$
x \cdot y= \begin{cases}f(x, y, a), & x \neq y \\ x, & x=y .\end{cases}
$$

We also say that the triple system $\left(Q \backslash\{a\}, B_{a}\right)$ is induced by $(Q \backslash\{a\}, \cdot)$.

An isomorphism from a Steiner system $\left(Q_{1}, B_{1}\right)$ with parameters $t, k, v$ onto a Steiner system $\left(Q_{2}, B_{2}\right)$ of the same type is a bijection $\varphi: Q_{1} \rightarrow Q_{2}$ which maps the $k$-tuples of $Q_{1}$ onto $k$-tuples of $Q_{2}$. An automorphism of $(Q, B)$ is an isomorphism of $(Q, B)$ onto itself. If $\varphi$ is an isomorphism from a Steiner quasigroup $\left(Q_{1}, \cdot\right)$ onto a Steiner quasigroup $\left(Q_{2}, *\right)$, then $\varphi$ is also an isomorphism from the corresponding $\operatorname{STS}\left(Q_{1}, B_{1}\right)$ of $\left(Q_{1}, \cdot\right)$ onto the corresponding $\operatorname{STS}\left(Q_{2}, B_{2}\right)$ of $\left(Q_{2}, *\right)$. Namely, if $\{a, b, c\} \in B_{1}$ then $\varphi(c)=\varphi(a \cdot b)=\varphi(a) * \varphi(b)$, meaning that $\{\varphi(a), \varphi(b), \varphi(c)\} \in B_{2}$. The same 
property also holds for $S Q S$. We use it to obtain an algebraic characterization of one class of homogenous Steiner quadruple systems.

The stated relations between Steiner systems and Steiner quasigroups are of great importance for both combinatorial and algebraic structures. Some properties are easier to be proved algebraicly, and others combinatorially. If we prove one in either way, then the corresponding property can be applied to the other structure. In our paper we use algebraic tools to obtain the desired combinatorial property.

\section{DESCRIPTION OF A ClaSS OF HoMOgenOUS SQS}

Given an $S Q S$, let $\beta$ denote the number of pairwise non-isomorphic derived triple systems. Obviously, $1 \leq \beta \leq v$, for any $\operatorname{SQS}(v)$. The least $v$ for which $\beta>1$ is 14 . There are 4 non-isomorphic $S Q S(14)$, and for two of them $\beta=1$, while for the other two $\beta=2$ (see [8]). It is clear that these are the only two possible values for $\beta$ since there are exactly two non-isomorphic STS(13).

Although infinite classes of $S Q S$ with $\beta \geq 2$ were constructed, as well as $S Q S(v)$ with $\beta \geq t$ for any positive integer $t$ ( $v$ much greater than $t$ ), the question of determination of $\beta$ for any given $S Q S$ is very far from solved. Moreover, no one as yet has found an order $v$ such that for every $k, 1 \leq k \leq v$, there is an $\operatorname{SQS}(v)$ having $\beta=k$.

An $S Q S$ is said to be homogenous if its value of $\beta$ is one, or equivalently if all of its derived triple systems are isomorphic. If all the DTS of an $\operatorname{SQS}(v)$ are pairwise non-isomorphic $(\beta=v)$, then the quadruple system is called heterogenous.

In what follows, we give an algebraic description of one class of homogenous Steiner quadruple systems.

Lemma. Let $(Q, f)$ be a finite Steiner 3-quasigroup and $\varphi$ be an automorphism of $Q$. Then for $a \in Q$, the derived triple systems $\left(Q \backslash\{a\}, B_{a}\right)$ and $\left(Q \backslash\{\varphi(a)\}, B_{\varphi(a)}\right)$ of the quadruple system induced by $(Q, f)$ are isomorphic. 
Proof. Let $(Q, B)$ be the $S Q S$ induced by $(Q, f)$ and define $\psi: Q \backslash\{a\} \rightarrow Q \backslash\{\varphi(a)\}$ by $\psi(u)=\varphi(u)$. Let $(Q \backslash\{a\}, \circ)$ and $(Q \backslash\{\varphi(a)\}, *)$ be the Steiner quasigroups which induce the triple systems $\left(Q \backslash\{a\}, B_{a}\right)$ and $\left(Q \backslash\{\varphi(a)\}, B_{\varphi(a)}\right)$ respectively. It is clear that $\psi$ is a bijection, since $\varphi$ is a bijection. If $u, v \in Q \backslash\{a\}$, and $u \neq v$, then

$$
\begin{aligned}
\psi(u \circ v) & =\psi(f(u, v, a))=\varphi(f(u, v, a)) \\
& =f(\varphi(u), \varphi(v), \varphi(a)) \\
& =\varphi(u) * \varphi(v)=\psi(u) * \psi(v) .
\end{aligned}
$$

The equality $\psi(u \circ u)=\psi(u)=\psi(u) * \psi(u)$ completes the proof.

Corrolary 1. Le $t(Q, f)$ be a finite Steiner 3-quasigroup with the property that for every $a, b \in Q$, there is an automorphism $\varphi$ such that $\varphi(a)=b$. Then the quadruple system induced by $(Q, f)$ is homogenous.

Note that the converse is false, and the smallest example of this is obtained for order $v=16$ (see [8]).

Theorem. Let $(Q, f)$ be a finite Steiner 3-quasigroup. Then the mapping $\varphi(x)=f(s, t, x)$ is an automorphism of $Q$ for each $s, t \in Q$, if and only if $f(a, b, f(u, v, w))=f(f(a, b, u), f(a, b, v), f(a, b, w))$ is an identity of $Q$.

Proof. For every $a, b, x, y, z \in Q$ and the automorphism $\varphi(x)=f(a, b, x)$, we have

$$
\begin{aligned}
f(f(a, b, x), f(a, b, y), f(a, b, z)) & =f(\varphi(x), \varphi(y), \varphi(z)) \\
& =\varphi(f(x, y, z))=f(a, b, f(x, y, z)) .
\end{aligned}
$$

Conversely, let $f(a, b, f(u, v, w))=f(f(a, b, u), f(a, b, v), f(a, b, w))$ be an identity of $Q$, and $s, t$ be arbitrary elements of $Q$. First we prove that the mapping $\varphi: Q \rightarrow Q$ defined by $\varphi(x)=f(s, t, x)$ is a bijection.

Since $f$ is a quasigroup operation,

$$
\varphi(x)=\varphi(y) \Rightarrow f(s, t, x)=f(s, t, y) \Rightarrow x=y,
$$


which means that $\varphi$ is injective.

Let $v \in Q$ and $u=f(s, t, v)$. Then

$$
\varphi(u)=f(s, t, u)=f(s, t, f(s, t, v))=v,
$$

by the fact that $(Q, f)$ is a Steiner 3-quasigroup. Hence, $\varphi$ is surjective.

The mapping $\varphi$ is a homomorphism, as a direct consequence of the identity. Namely, for $x, y, z \in Q$, we have

$$
\begin{aligned}
\varphi(f(x, y, z)) & =f(s, t, f(x, y, z))=f(f(s, t, x), f(s, t, y), f(s, t, z)) \\
& =f(\varphi(x), \varphi(y), \varphi(z)) .
\end{aligned}
$$

Corrolary2. Let $V$ be the variety of algebras with one ternary operation, defined by the identities

$$
\begin{aligned}
& f(x, x, y)=y \\
& f(x, y, z)=f(x, z, y)=f(y, x, z) \\
& f(x, y, f(x, y, z))=z \\
& f(a, b, f(x, y, z))=f(f(a, b, x), f(a, b, y), f(a, b, z)) .
\end{aligned}
$$

Then every finite algebra of $V$ induces a homogenous SQS.

Proof. The first three of the defining identities of $V$ determine the variety of Steiner 3-quasigroups, hence $V$ is its subvariety.

Let $(Q, f)$ be a finite algebra of $V$ and $(Q, B)$ be its induced quadruple system. We prove that for arbitrary elements $a, b \in Q$, the derived systems $\left(Q \backslash\{a\}, B_{a}\right)$ and $\left(Q \backslash\{a\}, B_{b}\right)$ of the quadruple system $(Q, B)$ are isomorphic. Let $\varphi: Q \rightarrow Q$ be the mapping defined by $\varphi(x)=f(a b)$. Then $\varphi$ is an automorphism of $Q$ by the preceding theorem. By the defining identities of $V$, we obtain $\varphi(a)=f(a, b, a)=b$. Then by using the result of the Lemma, we get that the derived systems $\left(Q \backslash\{a\}, B_{a}\right)$ and $\left(Q \backslash\{a\}, B_{b}\right)$ are isomorphic.

\section{EXAMPLES}

The algebraic representative of the unique $S Q S(8)$ satisfies the identity

$$
f(a, b, f(x, y, z))=f(f(a, b, x), f(a, b, y), f(a, b, z))
$$

implying that the $\operatorname{SQS}(8)$ is homogenous. 


\section{$1248 \quad 3567$ \\ 23581467 \\ 34681257 \\ 45781236 \\ 15682347 \\ 26781345 \\ 13782456}

Figure 1: The unique $S Q S(8)$

It is not necessary to check all the $8^{5}$ quintuples to get this result. Namely, this $S Q S$ is a member of the class of Steiner quadruple systems of orders $2^{n}$ (constructed by Kirkman) whose corresponding Steiner 3-quasigroups satisfy the identity

$$
f(x, y, f(z, y, t))=f(f(x, y, z), y, t)
$$

The subvariety of the variety of Steiner 3-quasigroups which is determined by the above identity is its unique minimal subvariety, i.e. it is the unique atom in the lattice of all subvarieties of the variety of Steiner 3-quasigroups (see [10]). Its algebras can easily be obtained from the class of Boolean groups. Given a Boolean group $(S,+)$, one needs only to define a ternary operation $f$ on $S$ by

$$
f(a, b, c)=a+b+c .
$$

The main question which arises from this discussion is whether this minimal subvariety is a proper subvariety of the variety $V$ of Corrolary 2, or the identities (1) and (2) are equivalent in the variety of Steiner 3-quasigroups.

The second "smallest" $S Q S$ is of order 10, and it is also unique (up to an isomorhism).

$$
\begin{aligned}
& 12451237 \quad 1358 \\
& 235623482469 \\
& 346734593570 \\
& 45784560 \quad 1468 \\
& 568915672579 \\
& 67902678 \quad 3680 \\
& 1780 \quad 37891479 \\
& 128948902580 \\
& 2390 \quad 1590 \quad 1369 \\
& 1340 \quad 12602470
\end{aligned}
$$

Figure 2: The unique $S Q S(10)$ 
It is cyclic, which means that it has an automorphism consisting of a single cycle of length 10. Such an $S Q S$ belongs to a class of the so called transitive $S Q S$, i.e. SQS whose automorphism group acts transitively on the elements. This class of $S Q S$ is precisely the class described in Corrolary 1. According to the Lemma, the $S Q S(10)$ is an example of a homogenous $S Q S$. However, (1) is not an identity of its corresponding Steiner 3-quasigroup:

$$
f(1,2, f(4,7,9))=f(1,2,1)=2 \text {, }
$$

but

$$
f(f(1,2,4), f(1,2,7), f(1,2,9))=f(5,3,8)=1 .
$$

This shows that the identity (1) provides sufficient, but not necessary condition for the induced $S Q S$ of a Steiner 3-quasigroup to be homogenous.

\section{CONCLusions}

Steiner systems and other combinatorial designs have attracted mathematicians with their uniform distribution of elements into sets for a long time. Since the first results, a huge progress in combinatorics is made, but yet, there are so many unanswered questions and open problems.

The algebraic approach in studying Steiner systems contributed a lot in their understanding in the past 40 years, and the related algebraic structures have become a non-separating part of the combinatorial research.

The summation of the efforts in resolving the problem of classification of Steiner quadruple systems according to the number of their pairwise nonisomorphic derived triple systems is a collection of particular results which are partial and unsystematic. The research of $S Q S$ with a minimal, and $S Q S$ with a maximal possible number of classes of isomorphic DTS, i.e. homogenous and heterogenous $S Q S$ is not completed, as well. It was conjectured that both types of $S Q S$ exist, for every order $v \geq 16$.

The result of this paper brings a small contribution to the research of homogenous $S Q S$, providing a nice description of a specific class of such $S Q S$. 
References

[1] Š. O. Aliev, Simmetričeskije algebry i systemy Štejnera, Dokl. Akad.Nauk SSSR 174 (1967), 511-513.

[2] B. Ganter, H. Werner, Equational classes of Steiner systems, Algebra Universalis 5 (1975), 125-140.

[3] L. Goračinova-Ilieva, (k,n)-Algebri, kvazigrupi i dizajni, Pedagoški fakultet "GoceDelčev" Štip, 2007

[4] H. Hanani, On quadruple systems, Can. J. Math. 12 (1960), 145-157.

[5] A. Hartman, K. T. Phelps, Steiner quadruple systems, in: J. H. Dinitz, D. R. Stinson (Eds.), Contemporary Design Theory, Wiley, New York, 1992, 205-240.

[6] P. Kaski, P. R. J. Ostergard, O. Pottonen, The Steiner quadruple systems of order 16, Journal of Combinatorial Theory, Series A, Vol. 113 (8) (2006), 1764-1770.

[7] T. P. Kirkman, On a problem in combinations, Cambridge and Dublin Math. J. 2 (1847), 191-204.

[8] C. C. Lindner, A. Rosa, Steiner quadruple systems - a survey, Discrete Math., 22 (1978), 147-181.

[9] K. T. Phelps, Some sufficient conditions for a Steiner triple system to be a derived triple system, Journal of Combinatorial Theory 20 (A) (1976), 393397.

[10] R. W. Quackenbush, Algebraic aspects of Steiner quadruple systems, in: Proc. Conf. Algebraic Aspects of Combinatotics, Toronto, 1975,

Congressus Numerantium XIII, Winnipeg 1975, 265-268.

[11] W. S. B. Woolhouse, Prize question 1733, Lady's and Gentleman's Diary, 1844

[12] H. L. de Vries, Some Steiner quadruple systems $S(3,4,16)$ such that all 16 derived systems $S(2,3,15)$ are isomorphic, Ars Combin., 24A (1987), 107 129.

${ }^{1)}$ Faculty of Informatics, FON University, Skopje, Macedonia

E-mail address: lidija.goracinova@fon.edu.mk

${ }^{2)}$ Faculty of Informatics, FON University, Skopje, Macedonia

E-mail address: emilija.kamceva@fon.edu.mk 